\title{
A Newly Recorded Spider from Japan, Phaeocedus braccatus (L. Ko:H) (Araneae: Gnaphosidae)
}

\author{
Takahide KAMURA ${ }^{1)}$
}

\author{
加村隆英 ${ }^{1)}$ ：日本新記録のワシグモ科の 1 種, \\ Phaeocedus braccatus (L. KocH)
}

\begin{abstract}
A gnaphosid species, Phaeocedus braccatus (L. KocH, 1866), is recorded from Nagano and Okayama Prefectures, Japan. The genus Phaeocedus is newly added to the Japanese fauna.
\end{abstract}

In 1980, I collected a spider of a certain species belonging to the family Gnaphosidae from Nagano Prefecture. Having a dorsal scutum on abdomen, this specimen seems to be an adult male. However, unfortunately both palps were broken off and missing. Although I suspected that this spider belongs to Phaeocedus braccatus (L. KocH, 1866) on the basis of the examination of its ocular area, margins of fang furrow and markings of abdomen, I was not convinced of the specific identification because of the lack of palps.

Recently I had an opportunity to examine a male gnaphosid spider collected by Mr. Kouichi NoJima from Okayama Prefecture. I identified this spider with Phaeocedus braccatus by the structure of its palp. Besides, I compared the two specimens from Nagano and Okayama Prefectures and recognized that they are conspecific with each other.

The genus Phaeocedus has never been recorded from Japan. Phaeocedus braccatus was well known in Europe (for example, TUlLGREN, 1946; LockeT \& Millidge, 1951; Grimm, 1985; RoBeRTs, 1985), and was recorded also from China (Hu \& WU, 1989) and eastern Siberia (Izmailova, 1989). In this paper, I report this species from Japan for the first time.

The abbreviations used in this paper are as follows: ALE, anterior lateral eye; AME, anterior median eye; MOA, median ocular area; $\mathrm{p}$, proventral; PLE, posterior lateral eye; PME, posterior median eye. Eye size means the length of long axis of an eye, but the measurement of posterior median eye was made at the horizontal level.

Before going further, I wish to express my sincere thanks to Mr. Kouichi NoJima, Okayama Pref., for offering an invaluable specimen.

1) Biological Laboratory, Otemon Gakuin University, 2-1-15, Nishi-Ai, Ibaraki, Osaka, 567 Japan

追手門学院大学生物学研究室 T567 茨木市西安威 2-1-15

Accepted July 17, 1995 
Phaeocedus braccatus (L. КосH, 1866)

(Figs. 1-3)

Drassus braccatus L. KocH, 1866, p. 97, pl. 4, fig. 63.

Phaeocedus braccatus: Simon, 1893, p. 370, fig. 327.——TUllgren, 1946, p. 127, figs. 36-37, pl. 21, figs. 269-271.—Locket \& Millidge, 1951, p. 106, figs. 50D, 55B, D, E.-RoewER, 1954, p. 428.-BonNET, 1958, p. 3495-_-GrimM, 1985, p. 158, figs. 1b, 179-183.-—RoBerTs, 1985, vol. 1, p. 70, fig. 25c, vol. 3, pl. 23.—-PLATNICK, 1989, p. 481; 1993, p. 663.——Hu \& Wu, 1989, p. 281, fig. 226 (3-4)._-Izmailova, 1989, p. 108, figs. 92-93.

Description (based on $10^{\text {T }}$ from Okayama Pref.). Measurements (in mm). Body length 4.23. Carapace length 1.88, width 1.38. Abdomen length 2.35, width 1.40. Length of legs as shown in Table 1. Eye sizes: AME 0.11, ALE 0.12, PME 0.12, PLE 0.11. Distances between eyes: AME-AME 0.06, AME-ALE 0.01, PMEPME 0.04, PME-PLE 0.07, ALE-PLE 0.04. MOA anterior width 0.26, posterior width 0.28 , length 0.30 . Clypeus height 0.12 .
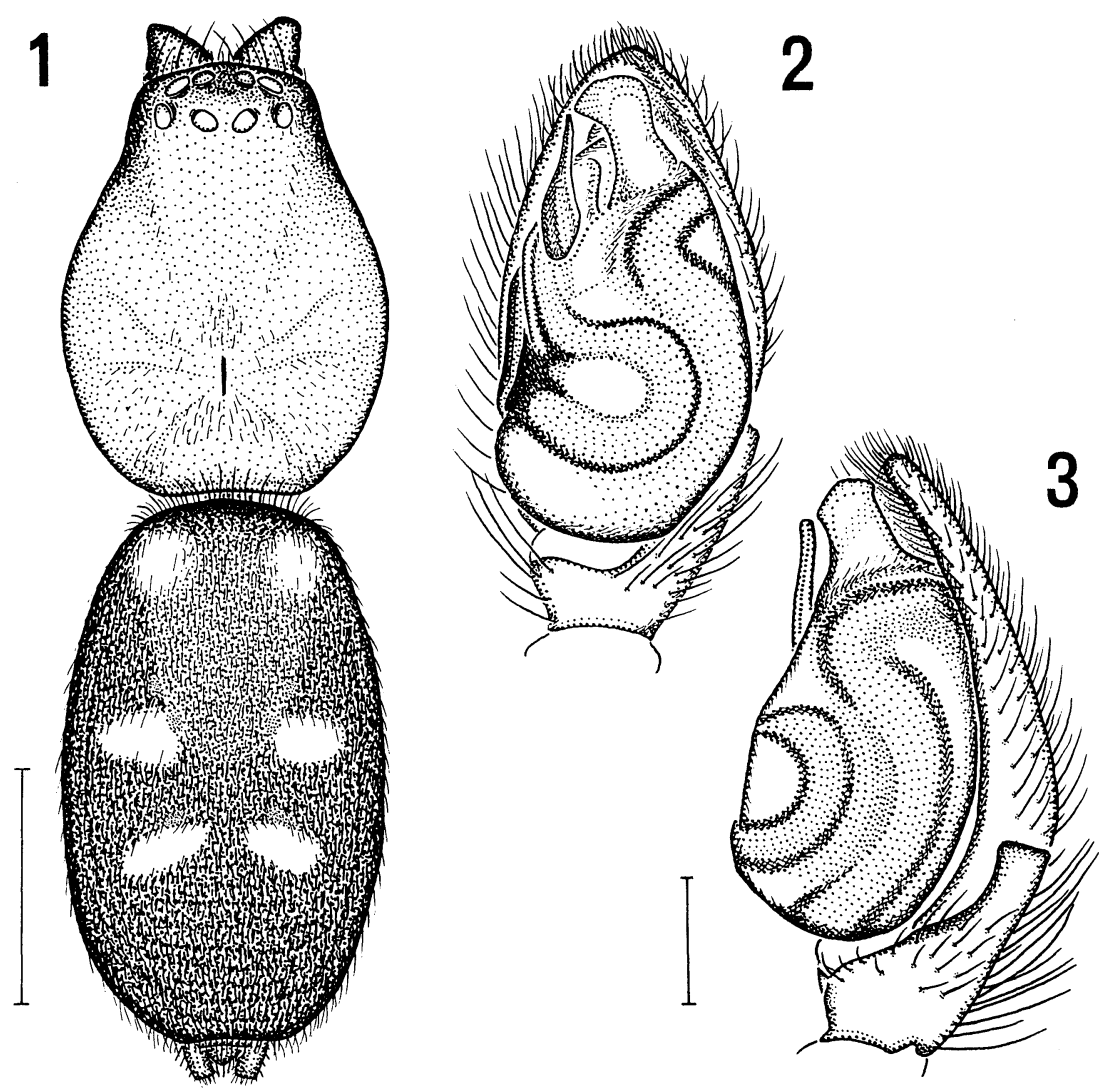

Figs. 1-3. Phaeocedus braccatus (L. KocH, 1866) (Okayama Pref.)._-1. Male body, dorsal view. 2. Left male palp, ventral view. 3. Same, retrolateral view. (Scales: 1, $1.0 \mathrm{~mm} ; 2-3,0.2 \mathrm{~mm}$.) 
Table 1. Measurements of legs of Phaeocedus braccatus (L. KocH, 1866) (ơ' in mm).

\begin{tabular}{lcccccc}
\hline Leg & Femur & Patella & Tibia & Metatarsus & Tarsus & Total \\
\hline I & 1.25 & 0.70 & 0.96 & 0.84 & 0.64 & 4.39 \\
II & 1.12 & 067 & 0.82 & 0.73 & 0.59 & 3.93 \\
III & 1.00 & 054 & 0.64 & 0.68 & 0.48 & 3.34 \\
IV & 1.32 & 0.70 & 0.98 & 1.06 & 0.55 & 4.61 \\
\hline
\end{tabular}

Ventral spines on legs I and II. Tibiae: I and II $0-0-0$ or $0-0-1 p$; metatarsi: I and II 0-0-0.

Anterior eye row slightly recurved and posterior eye row slightly procurved as seen from above. Chelicera with a carina on promargin of fang furrow, and no tooth on retromargin. Male palp (Figs. 2-3): median apophysis distinct, conductor membranous, retrolateral tibial apophysis truncated apically.

Color. Carapace, chelicerae, palps, endites and labium dark reddish brown. Sternum dusky yellow. Coxae I, trochanters I, and femora I and II dark reddish brown, the other segments of legs I and II and all segments of legs III and IV dusky yellow. Abdomen blackish brown, with three pairs of white markings on dorsum.

Variation. Measurements of $10^{7}$ from Nagano Pref.: Body length $5.03 \mathrm{~mm}$; carapace length $2.20 \mathrm{~mm}$, width $1.58 \mathrm{~mm}$.

Specimens examined. 10 $10^{\top}$, Suehiro, Suwa-shi, Nagano Pref., 26. VII. 1980 (T. Kamura). 10', Ôtani-tôge, Okutsu-cho, Tomata-gun, Okayama Pref., 11. VII. 1993 (K. NoJima).

Distribution. Japan (Honshu); Palearctic.

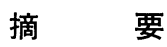

ワシグモ科の Phaeocedus braccatus (L. KocH，1866) タソガレトンビグモ（新称）を日 本新記録種として報告した.Phaeocedus タソガレトンビグモ属（新称）のクモが日本から 記録されたのは初めてである。

\section{References}

BonNet, P., 1958. Bibliographia araneorum, 2(4), pp. 3027-4230. Toulouse.

Grimm, U., 1985. Die Gnaphosidae Mitteleuropas (Arachnida, Araneae). Abh. naturw. Ver. Hamburg, (NF), 26: 1-318.

Hu, J. L., \& W. G. Wu, 1989. Spiders from Agricultural Regions of Xinjiang Uygur Autonomous Region, China. 435 pp. Shandong Univ. Publ. House, Jinan. (In Chinese.)

Izmailova, M. V., 1989. Fauna of Spiders of South Part of Eastern Siberia. 184 pp. State Univ. Publ., Irkutsk. (In Russian.)

Kосн, L., 1866. Die Arachniden. Familie der Drassiden, 1-6. 304 pp., 12 pls. Nürnberg. Locket, G. H., \& A. F. Millidge, 1951. British Spiders, 1. x+310 pp. Ray Society, London.

Platnick, N. I., 1989. Advances in Spider Taxonomy 1981-1987. 673 pp. Manchester Univ. Press, Manchester and New York.

1993. Advances in Spider Taxonomy 1988-1991. 846 pp. New York Entomol. Soc. and Amer. Mus. Nat. Hist., New York. 
Roberts, M. J., 1985. The Spiders of Great Britain and Ireland, 1, 229 pp.; 3, 256 pp. Harley Books, Essex.

Roewer, C. F., 1954. Katalog der Araneae, 2 (a). 923 pp. Bruxelles.

Simon, E., 1893. Histoire Naturelle des Araignées, 1(2), pp. 257-488. Paris.

Tullgren, A., 1946. Svensk Spindelfauna, 3. 141 pp., 21 pls. Stockholm. 